\title{
Review
}

\section{Genetic and Non-Genetic Basis of Essential Hypertension: Maladaptation of Human Civilization to High Salt Intake}

\author{
Kiyoshi Kurokawa, and Toshihiro Okuda*
}

\begin{abstract}
The relation between salt intake and hypertension has long been controversial. Available evidence provides conflicting results. However, biological and evolutionary insights into the structure and principle function of the kidney and the blood pressure sustaining machinery, with the extracellular fluid volume and the renin-angiotensin system acting as a central player, clearly indicate that salt has been a precious commodity in the terrestrial animal kingdom and that essential hypertension may be a consequence of the kidney's maladaptation to excess salt intake, which is specific to recent human civilization. This review provides a hypothesis to explain how and why hypertension develops and attempts to define the roles of genetic, non-genetic, and environmental factors in its pathogenesis. (Hypertens Res 1998; 21: 67-71)
\end{abstract}

Key Words: hypertension, kidney, salt, juxtaglomerular apparatus, human civilization

The human kidneys, consisting of approximately 2 million nephrons, receive $1 \mathrm{l} / \mathrm{min}$ of blood flow, the highest blood flow per organ weight in the body. When the blood flows through the glomeruli, a highly efficient filtration system, each kidney generates $100 \mathrm{ml} / \mathrm{min}$ of urine. Approximately 99\% of this glomerular filtrate is reabsorbed along the tubules, a highly organized and regulated epithelial transporting system. Why is such a process necessary? The principal function of the kidney is to maintain the homeostasis of our milieu interieur, or the extracellular fluid (ECF), despite wide variations in and ranges of daily fluid and electrolyte intake. The range of daily fluid and electrolyte intake that causes no significant disturbances in the milieu interieur is quite large: water intake could range from 0 to $30 \mathrm{l} / \mathrm{d}$; salt, 0 to $1,000 \mathrm{mEq} / \mathrm{d}$; potassium, 0 to $700 \mathrm{mEq} / \mathrm{d}$, and so on. This wide allowance in the intake of fluids and electrolytes is due to a high glomerular filtration rate (GFR) of $140 \mathrm{~d}$, with approximately $99 \%$ reabsorption of the glomerular filtrate. A patient with a GFR of $10 \%$ of normal would have to have a much lower intake of fluid and electrolytes, because a higher intake would cause pathological changes in the milieu interieur. It is this remarkable kidney function of high filtration and high reabsorption that allows us great flexibility in our daily fluid and electrolyte intake. A detailed discussion of some of the issues outlined in this overview has appeared elsewhere $(1,2)$.

\section{Autoregulation of Renal Blood Flow}

To maintain a high GFR despite possible fluctuations in blood pressure in a terrestrial environment with $1.0 \mathrm{G}$ gravity, the kidney must maintain a constant blood supply and GFR. Table 1 summarizes the principal functions of the kidney (2). Constancy of renal blood flow is achieved with a two-component system: a myogenic response of the afferent artery (AA), and tubuloglomerular feedback (TGF) by the juxtaglomerular apparatus (JGA). In response to changes in renal perfusion pressure, vascular smooth muscles of the AA respond to maintain the down-stream perfusion pressure so that the renal blood flow remains constant, i.e., myogenic response. The very last portion of the $\mathrm{AA}$ at the entrance to the glomerulus is regulated principally by TGF; this portion contracts or relaxes in response to an increase or decrease, respectively, in macula densa chloride $\left(\mathrm{Cl}^{-}\right)$delivery (3). Moreover, Holstein-Rathlou and colleagues $(4,5)$ have reported oscillations with a frequency of approximately $20 \mathrm{~s}$ of the distal tubular fluid $\left[\mathrm{Cl}^{-}\right]$just beyond the macula densa and of the proximal tubule pressure, reflecting single nephron glomerular filtration rates (SNGFR) of the same tubule. This oscillation is driven by TGF at the entrance of the AA to the glomerulus. These observations clearly indicate that the apparent constancy of the SNGFR is maintained by the TGF.

From the Department of Internal Medicine, Tokai University School of Medicine, Isehara, Japan, and * Department of Internal Medicine, Faculty of Medicine, the University of Tokyo, Tokyo, Japan.

Address for Reprints: Kiyoshi Kurokawa, M.D., Dean and Professor of Medicine, Tokai University School of Medicine, Isehara, Kanagawa 259-1193, Japan.

Received March 14, 1998. 
Table 1. Principal Functions of the Kidney

1. To maintain homeostasis of the milieu interieur with diverse and constantly changing inputs of nutrients, fluids, and minerals. The route of input is principally through the mouth.

2. For homeostasis of the ECF to be maintained, the kidney has to excrete in the urine quantities of fluid and electrolytes precisely matched to their input to the body. Urine is the only route that can excrete fluid and electrolytes in a regulated manner.

3. To achieve this goal, there must be specific sensing mechanisms for specific changes in each of the constituents of the $\mathrm{ECF}$, such as volume, toxicity, potassium concentration, and calcium concentration.

4. Regardless of the sensing mechanisms, however, it is the kidney that responds to these sensing mechanisms to maintain homeostasis of the ECF.

5. For the kidney to effectively perform this principal function, it must have high glomerular filtration and almost $99 \%$ tubular reabsorption to allow maximal stability to the homeostatic system.

6. GFR is high relative to ECF volume; thus, an increase in GFR could impose an immediate threat to life due to a rapid fall in ECF volume.

7. Therefore, constancy of a high GFR and RPF must be met in response to changes in systemic blood pressure and renal perfusion pressure, i.e., autoregulation.

\section{Autoregulation and Maintenance of Single Nephron Glomerular Filtration Rates}

What is the possible physiological importance of autoregulation of renal glomerular plasma flow? Consider a situation where salt intake is very low, perhaps less than $2 \mathrm{~g} / \mathrm{d}$. The systemic blood pressure and renal perfusion pressure will drop. A decrease in AA resistance $\left(R_{A}\right)$ due to the myogenic response of AA and also due to TGF at the entrance to the glomerulus maintains glomerular plasma flow (GPF). A drop in ECF volume and systemic blood pressure maximally activates the reninangiotensin system (RAS). Maximal activation of the RAS leads to a preferential increase in efferent arteriolar resistance $\left(\mathrm{R}_{\mathrm{E}}\right)$ by angiotensin II (AII) to maintain glomerular capillary pressure, thus maintaining the SNGFR. This process allows a wide range of the daily intake of fluids and minerals, despite a low salt intake (6).

\section{Phylogenic Considerations of Juxtaglomerular Apparatus}

It is thought that the JGA and TGF appear in the nephrons of amphibians, reptiles, birds, and mammals $(7,8)$. We could therefore envision the functional roles of JGA or TGF from an evolutionary perspective. Simply compare the milieu interieur of the sea fish, teleost, to that of humans. The basic constituents of intracellular and extracellular fluids are comparable: thus, the ECF, or milieu interieur, is isotonic saline, and potassium is the major cation of the intracellular fluids for both teleosts and humans. When a fish ingests its meal, the salt it absorbs has a higher concentration than that of the milieu interieur, i.e., $0.9 \%$ saline in ECF vs. $3.5 \%$ $\mathrm{NaCl}$ in sea water. Thus, the teleost dose not need salt, but rather needs salt-free water. The milieu interieur of the teleost is maintained primarily by the gills and the kidney has only an ancillary role.

In contrast, there is no guarantee of salt intake for terrestrial creatures. In fact, the availability of salt is, in general, very limited for terrestrial life, since the main sources of food stuff in the natural environment are plants and animal meats. Nonetheless, it is necessary to maintain a high GFR to maintain the milieu interieur in response to a wide range of fluid and electrolyte intake. This is probably why the JGA first appeared in amphibians, and it is likely that the JGA was an essential component of the nephron in order for organisms to live on lands, where a high GFR must be maintained with a minimal salt intake. It should be noted that blood pressure must rise in terrestrial life to ensure that the blood supplies oxygen throughout the body system in $1.0 \mathrm{G}$ gravity (9). The blood pressure rise helps to develop a high GFR.

\section{Renal Cross Transplantation in Rat Models of Hypertension}

Results of renal cross transplantation studies in an experimental model of hypertension in the rat strongly indicate that the kidney has a primary role in the genesis of hypertension (10). Thus, available data clearly indicate that the kidney determines whether or not hypertension eventually develops. Similar results have also been obtained from studies in humans. In chronic dialysis patients with end stage renal failure due to severe hypertension, successful kidney transplantation from a normotensive, healthy donor often leads to the disappearance of hypertension (11). These observations are consistent with the notion that it is the kidney that dictates the development of hypertension.

\section{Aberrant Tubuloglomerular Feedback as an Intrarenal Mechanism Responsible for Abnormal Salt Excretion}

There are numerous reviews and monographs on the role of the kidney in the genesis of hypertension. In particular, two recent reviews address the critical role of the kidney. One role proposed by 
Laragh and colleagues emphasizes the presence of a subset of nephrons that secrete inappropriately increased amounts of renin for any given salt intake (12). Another hypothesis, proposed by Brenner and colleagues, indicates that a decreased number of nephrons, and thus a less effective glomerular filtration surface, is associated with the development of hypertension (13). This hypothesis is also persuasive.

A third hypothesis that we advance is that an abnormal JGA or resetting of the TGF might be involved in the development of hypertension (2). Available data indicate similarities in the TGF response of spontaneously hypertensive rats (SHR) and Wistar Kyoto rats (WKY) and that of normal rats with and without AII. Thus, with a given salt intake, TGF is more activated in SHR than in WKY. This difference is quite similar to that between normal rats with and without AII $(14,15)$. The data suggest that the TGF of SHR is behaving as if TGF is more sensitive to AII for any given salt intake or ECF volume. Thus, in response to a saline load, the RAS will be inhibited because of volume expansion, and the TGF curve will become either less activated or inhibited; at the same time, distal $\mathrm{Cl}^{-}$delivery is increased by the saline load, but the inhibition of TGF increases the GFR, and saline is effectively excreted in the urine. In hypertensive subjects, however, this TGF inhibition is incomplete or aberrant; thus, in response to increased distal $\mathrm{Cl}^{-}$delivery, the GFR might go unchanged or even drop, rather than increase, leading to volume expansion with a slower urinary excretion of loaded saline. This is a characteristic response in hypertensive patients or even some normotensive subjects with a family history of essential hypertension $(16,17)$. This series of changes in response to salt loading eventually leads to hypertension with increased peripheral resistance if excess salt intake continues. Thus, in hypertensive patients, inability of the kidneys to excrete salt must be responsible for the development and maintenance of hypertension (18). The hypothesis of Laragh and colleagues is consistent with the abnormal TGF governed by the RAS, particularly in superficial nephrons.

\section{Lower Nephron Number as an Intrarenal Mechanism Responsible for Abnormal Salt Excretion}

Another renal abnormality that might underlie the genesis of hypertension is a lower number of nephrons or a lower glomerular filtration surface area, as proposed by Brenner and colleagues (13). According to this hypothesis, hypertension will develop in individuals with a high salt intake and a lower number of nephrons since in such individuals the ability of the kidney to eliminate excess salt would be impaired as compared with a normal subject with a sufficient number of nephrons. Data supporting such a hypothesis have been obtained from both clinical and experimental studies. Comparable models in which hypertension also develops would be congenital or acquired forms of a reduction in, or ablation of, renal mass.
If this hypothesis is correct, then in persons with a family history of essential hypertension, genetic defects must be somehow involved in the growth and development of the kidney during fetal life, since the number of nephrons would be determined by the time of birth and would not increase significantly thereafter. Such genetic abnormalities have not yet been identified. Nonetheless, it is important to note that there are other conditions that might affect the nephron number in individuals born after a full-term pregnancy. Available evidence suggests a good correlation between birth weight and later development of hypertension; indeed, birth weight is inversely correlated with blood pressure in childhood, adolescence, and adulthood, and this correlation is amplified with increasing age (19-21).

According to Brenner, this correlation might be due to a lower number of nephrons, which is determined by low birth weight and due principally to intrauterine protein malnutrition. Such conditions might also cause hypertension with the high salt intake of our culture in those who were in utero during poor nutritional conditions, such as those associated with poverty, low socioeconomic conditions, and war. Hypertension might develop in siblings because of similar intrauterine nutrition provided by their mother. Nonetheless, it is clear that hypertension is not necessarily a genetically determined event. It is thus important to note that such conditions should be regarded as a non-genetic cause of hypertension, even in the presence of a "family history."

\section{Structural Alterations in the Kidney in Essential Hypertension}

Whatever pathogenic factors underlie essential hypertension, the dominant and most characteristic renal abnormality in essential hypertension has been documented to be afferent arteriolar hyalinosis, which is directly related to both the severity and duration of hypertension (22). The glomeruli and postglomerular structures might be subject to ischemic changes. Such pathologic changes in the kidney are consistent with aberrant TGF, since TGF is associated with increased afferent arteriolar resistance. Nonetheless, as hypertension progresses in severity and duration, renal arterioles and the glomeruli begin to show direct influences of the high perfusion pressure as well as ischemic changes.

In contrast, if the lower number of nephrons is the prevailing cause of essential hypertension, one might expect to see the presence of hypertrophic glomeruli with focal sclerosis in the glomerular tufts in early hypertension, early renal lesions typically seen in remnant kidney, a model of oligonephronia. This must be carefully examined, particularly in hypertensive subjects with good documentation of uterine malnutrition and low birth weight $(20,21)$. In any event, a detailed examination of early renal lesions in subjects with essential hypertension might reveal a relative prevalence of distinct pathogenic factors, which might vary depending upon region, race, historical events, and other factors. 
Table 2. Principal Functions of the Kidney and the Genesis of Hypertension

1. The function of the kidneys is to maintain a high GFR even when faced with a low ECF volume, a condition essential for a stable milieu interieur that allows great flexibility in fluid and mineral intake.

2. This stability has been acquired during the transition from the high salt environment of sea water to terrestrial life, where salt intake is not guaranteed.

3. The JGA apparatus is critical for this function.

4. Altered JGA function or TGF is a cause of hypertension and becomes manifest only when challenged with a high salt intake.

5. High salt intake is a behavior that contradicts the principal structure and function of the kidney, i.e., a critical adaptation necessary for transition from a sea water environment to a terrestrial environment.

6. Hypertension is the price some of us must pay for the high salt intake in our culture.

\section{Salt Intake in Human Civilization}

According to the hypothesis outlined here, hypertension will not develop where salt intake is very low. Epidemiological studies indicate that this is indeed the case. People with very low salt intake, e.g., Papua New Guineans and Yanomamo Indians in the Amazon, do not develop hypertension, and blood pressure does not rise as they get older (23).

We must realize that only humans have acquired the habit of consuming excess salt (23). Due to this habit of high salt intake, some will become hypertensive because they cannot adapt appropriately to a chronic excess salt intake because of a disorder in the TGF. In a natural environment, mammals do not constantly consume excess salt. The salt intake of carnivores is 20 to $40 \mathrm{mEq} / \mathrm{d} / 60$ $\mathrm{kg}$ body weight, but that of herbivores is less than $10 \mathrm{mEq} / \mathrm{d} / 60 \mathrm{~kg}$ body weight. Thus, terrestrial mammals in a natural environment are in a state of chronic volume depletion with a highly activated RAS.

Analyses of Paleolithic nutrition suggest that salt intake by human beings, Homo sapiens, was 30 $\mathrm{mmol} / \mathrm{d}$ at most, with a potassium intake of 500 to $700 \mathrm{mmol} / \mathrm{d}(24)$. The development of agriculture approximately 10,000 years ago did not change this salt intake when vegetable foods came to make up $90 \%$ of the diet. Thus, throughout our civilization, salt has been a valuable commodity and not an item in abundance. Abundant evidence suggests the importance of salt in our culture and civilization. Interested readers are referred to excellent monographs by Denton (23) and Astrap (25) and a recent brief review by Ritz (26).

\section{Genetic, Non-Genetic, and Environmental Factors of Hypertension}

Our body system has evolved through years of evolution accompanied by genetic mutations. The kidneys and the blood pressure maintenance system are no exception. Thus, our body system is genetically adapted to the salt depletion of the terrestrial environment. "Industrialized civilizations" have introduced an excessive salt intake, however, leading to a condition called essential hypertension or a condition of maladaptation to chronic high salt intake in a subset of people. Obviously, the last few centuries have not provided enough time for our body to genetically adapt to such excessive salt intake. In fact, some genetic abnormalities responsible for hypertension, such as a mutation in the distal $\mathrm{Na}^{+}$channels leading to increased $\mathrm{Na}^{+}$reabsorption in Liddle syndrome, would have been advantageous for survival in a natural environment with a low salt intake. This abnormality has been shown to be a cause of hypertension only in recent years because of our high salt intake as a culture. Indeed, mutations that have an opposite effect on the same $\mathrm{Na}^{+}$channel, i.e., decreased distal $\mathrm{Na}^{+}$ reabsorption, would have caused severe dehydration in children who inherited the trait, a condition quite disadvantageous for survival in a natural 'primitive' environment with low salt availability. Thus, such mutations of the $\mathrm{Na}^{+}$channel could not be conferred to subsequent generations. This is the reason why we do not see such mutations. These are the bases for genetic and environmental factors in the genesis of hypertension.

Moreover, as observed in the case of oligonephronia, maternal protein malnutrition can cause hypertension in offsprings in a culture with high salt intake. This mechanism is not genetically determined, but might appear in the siblings, and thus can be viewed as "hypertension with a positive family history."

In any event, the differences among the three hypotheses presented here on the intrarenal mechanisms of essential hypertension could be viewed from slightly different perspectives. Two of the hypotheses, one by Laragh proposing the presence of a subset of nephrons with unsuppressed renin secretion for a given volume, and the other by Brenner, proposing a lesser number of nephrons, both explain the mechanism of how the kidney is unable to appropriately excrete excess salt, leading to development of hypertension. Abnormal JGA function or an abnormal TGF setting as a cause of hypertension likewise explains as well as the other two hypotheses how the kidney is unable to excrete salt intake, but it also explains why such abnormalities could have been present in humans. As proposed here, the kidney must have acquired, during the evolution of life and the transition from the sea to the land, the ability to maintain a high GFR in the face of a very low salt intake. Thus, high salt intake was never expected at the genetic level during 
human evolution. Stated differently, genetic abnormalities associated with essential hypertension would have been beneficial for survival in a natural environment with a low salt intake and were therefore preserved for many generations, only to become manifest in recent centuries because of the high salt intake of our culture (Table 2).

\section{References}

1. Kurokawa K, Okuda T: Calcium activated chloride conductance of mesangial cells. Kidney Int 1990; 38 (Suppl 30): S48-S50.

2. Kurokawa K: Kidney, salt, and hypertension: how and why. Kidney Int 1996; 49 (Suppl 55): S46-S51.

3. Ito S, Carretero OA: An in vivo approach to the study of macula densa mediated glomerular hemodynamics. Kidney Int 1990; 38: 1206-1210.

4. Holstein-Rathlou NH, Marsh DJ: Oscillations of tubular pressure, flow, and distal chloride concentration in rats. Am J Physiol 1990; 256: F1007-F1014.

5. Holstein-Rathlou NH: Oscillation and chaos in renal blood flow control. J Am Soc Nephrol 1993; 4: 12751287.

6. Badr KF, Ichikawa I: Prerenal failure: a deleterious shift from renal compensation to decompensation. $N$ Engl J Med 1988; 319: 623-629.

7. Persson BE, Persson AEG: The existence of a tubuloglomerular feedback mechanisms in the Amphiuma nephron. Pflugers Arch 1981; 391: 129-134.

8. Brown JA, Rankin JC, Yokota SD: Glomerular haemodynamics and filtration on single nephrons of non-mammalian vertebrates, in Brown JA, Balment RJ, Tankin JC (eds): New insights in vertebrate kidney function. Society for Experimental Biology Seminar Series 52, Cambridge, Cambridge University Press, 1993, pp1-44.

9. Schmidt-Nielsen K: Animal physiology. 4th ed., Cambridge, Cambridge University Press, 1990, pp299-390.

10. Rettig R, Unger $\mathrm{T}$ : The role of the kidney in the aetiology of hypertension. Trends Pharmacol Sci 1991; 12: 243-245.

11. Curtis JJ, Luke RG, Dustan HP, et al: Remission of essential hypertension after renal transplantation. $N$ Engl J Med 1983; 309: 1009-1015.

12. Sealey JE, Blumenfeld JD, Bell GM, Pecker MS,
Sommers SC, Laragh JH: On the renal basis for essential hypertension: nephron heterogeneity with discordant renin secretion and sodium excretion causing a hypertensive vasoconstriction-volume relationship. J Hypertens 1988; 6: 763-777.

13. Brenner BM, Garcis DL, Anderson S: Glomeruli and blood pressure: less of one, more the other? Am J Hypertens 1988; 1: 335-347.

14. Arendshorst WJ: Altered reactivity of tubuloglomerular feedback. Annu Rev Physiol 1987; 49: 295317.

15. Briggs JP, Schnermann J: The restoration of tubuloglomerular feedback in volume-expanded rats by angiotensin II. Am J Physiol 1990; 259: F565-F572.

16. Grim CE, Luft FC, Miller JZ, Brown PL, Gannon MA, Weinberger MH: Effects of sodium loading and depletion in normotensive first-degree relatives of essential hypertensives. J Lab Clin Med 1979; 94: 764-771.

17. Hollenberg NK, Moore T, Shoback D, Redgrave J, Rabinowe S, Williams GH: Abnormal renal sodium handling in essential hypertension. Am J Med 1986; 81: $412-418$.

18. Guyton AC: Dominance role of kidney and accessory role of whole-body autoregulation in the pathogenesis of hypertension. Am J Hypertens 1989; 2: 575585.

19. Barker DJP, Bull AR, Osmond C, Simmonds SJ: Fetal and placental size and risk of hypertension in adult life. Br Med J 1990; 301: 259-262.

20. Barker DJP, Winter PD, Osmond C, Margetts B, Simmonds SJ: Weight in infancy and death from ischemic heart disease. Lancet 1989; 2: 577-580.

21. Law CM, de Swiet M, Osmond C, et al: Initiation of hypertension in utero and its amplification throughout life. Br Med J 1993; 306: 24-27.

22. Sommers SC, Melamed J: Renal pathology of essential hypertension. Am J Hypertens 1993; 3: 583-587.

23. Denton D: The hunger for salt. Berlin, Heidelberg, New York, Tokyo, Springer Verlag, 1984.

24. Eaton SB, Konner M: Paleolithic nutrition. $N$ Eng $J$ Med 1985; 312: 283-289.

25. Astrap P: Salt and water in culture and medicine. Copenhagen, Munksgaard, 1993.

26. Ritz E: The history of salt-aspects of interest to the nephrologist. Nephrol Dial Transplant 1996; 11: 969975. 